\title{
Das Themenportal Erster Weltkrieg der Bibliothek für Zeitgeschichte
}

Nachdem der Erste Weltkrieg in Forschung und Öffentlichkeit jahrzehntelang im Schatten des Zweiten Weltkrieges stand, lenkt das Weltkriegsgedenkjahr 2014 nun in einmaliger Weise die Aufmerksamkeit auf die viel beschworene "Urkatastrophe des 20. Jahrhunderts". 100 Jahre Erster Weltkrieg - dieser Jahrestag ist für die Bibliothek für Zeitgeschichte (BfZ) von besonderer Bedeutung, schließlich wurde sie selbst 1915 als Sammlung über den Krieg gegründet. Die Bibliothek besitzt heute bedeutende Bestände zum "Großen Krieg". Hierzu zählen neben Büchern und Broschüren unter anderem Zeitschriften, Zeitungen, Plakate, Flugblätter, Fotos, Postkarten, Tagebücher und Briefe. Gleichzeitig ist der Erste Weltkrieg - neben dem Zweiten Weltkrieg und der Marinegeschichte - nach wie vor das wichtigste Themengebiet, zu dem die BfZ Material führt. Sie ist daher weltweit ein wichtiger Anlaufpunkt für Forscher und Publizisten. Aus dem zentralen Stellenwert des Ersten Weltkrieges entstand in der BfZ die Idee, der Wissenschaft, den Schulen sowie der Öffentlichkeit zum Weltkriegsgedenkjahr 2014 in Form eines Themenportals ein neuartiges digitales Informationsangebot zum Ersten Weltkrieg zur Verfügung zu stellen.

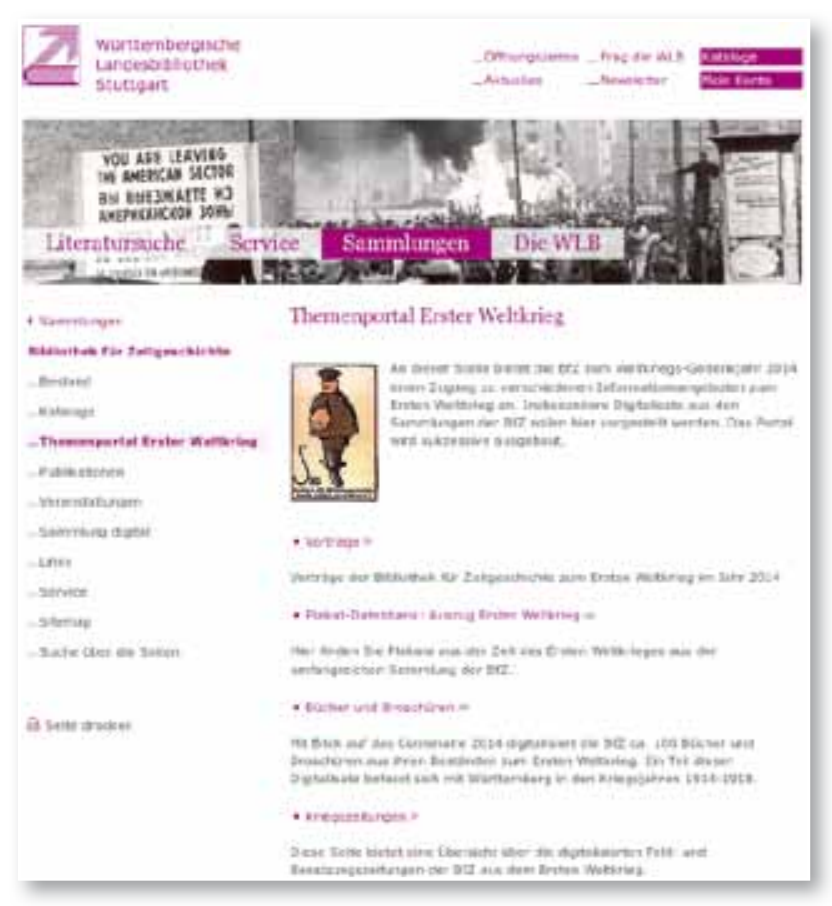

Abb. 1: Startseite des Themenportals auf der Homepage der WLB

\section{Ziele des Themenportals}

Für zahlreiche Medien der Präsenzbestände der $\mathrm{BfZ}$ ist es bisher notwendig, dass der Nutzer sowohl Findmittel als auch Bestände vor Ort einsieht. Nur ein kleiner Teil der Sondersammlungen zum Ersten Weltkrieg war bislang online recherchierbar. Die wenigen digitalen Angebote waren über die Homepage verstreut.

Vor diesem Hintergrund lag es nahe, eine zentrale Online-Präsenz der BfZ zum Thema Erster Weltkrieg aufzubauen, die alle digitalen Angebote der BfZ zum Ersten Weltkrieg bündelt und an zentraler Stelle auf der Homepage dem Nutzer präsentiert. Neben Hinweisen auf das Veranstaltungsangebot zum Ersten Weltkrieg enthält das "Themenportal Erster Weltkrieg" (Abb. 1) Rechercheinstrumente zu den verschiedenen Medienarten. Außerdem werden kontinuierlich Teile der gemeinfreien Bestände digitalisiert, um sie unabhängig vom Leihverkehr nutzbar zu machen. Damit kommt die Bibliothek den Benutzerwünschen nach ortsunabhängiger Recherche und Einsichtnahme entgegen und schont gleichzeitig ihre wertvollen Bestände. Die Angebote verweisen zudem auf die großen Materialsammlungen vor Ort und erhöhen so den Bekanntheitsgrad der Sammlungen.

Um die Auffindbarkeit des Themenportals zu optimieren, ist es direkt auf der Internetseite der BfZ angesiedelt. Dies hat den praktischen Nebeneffekt, dass eine möglichst kurze URL entsteht, die nur wenig Platz braucht, wenn sie als Link zitiert wird.

\section{Die Elemente des Portals}

Das Themenportal wurde im Sommer 2013 eingerichtet und seitdem ständig ausgebaut. Zum jetzigen Zeitpunkt umfasst es folgende Elemente, die auf Unterseiten verlinkt sind:

- Vorträge

- Plakat-Datenbank: Auszug Erster Weltkrieg

- Bücher und Broschüren

- Kriegszeitungen

- Tagebücher und Briefe 
- Rationierungsmarken-Datenbank: Ausschnitt Erster Weltkrieg

- Digitale Dokumentensammlung für Nutzer der WLB

\section{Vorträge}

An dieser Stelle informieren wir über die Vorträge der BfZ zum Ersten Weltkrieg. In den Jahren 20142018 wird sich die Vortragsreihe der Bibliothek schwerpunktmäßig den verschiedenen Aspekten des Krieges widmen. Die Reihe wurde eröffnet mit einem Gespräch mit Christopher Clark, dessen Buch "Die Schlafwandler"2 über die Vorgeschichte des Ersten Weltkrieges zeitweilig auf Platz 1 der Spiegel-Bestsellerliste rangierte. Clarks Buch hat seit seiner Übersetzung ins Deutsche eine neue Debatte über die Ursachen des Ersten Weltkrieges und die deutsche Schuld angestoßen und wird in zahlreichen Feuilleton-Beiträgen zitiert.

Im weiteren Verlauf des Jahres 2014 wird das Jahr 1914 im Fokus der Reihe stehen, 2015 dann das Jahr 1915. Für 2014 sind bisher folgende Vorträge geplant:

17.7.2014: Dr. Alex Watson: „Mit unerhörter Brutalität". Russische Gräueltaten in Ostpreußen, 1914-1915.

Alexander Watson, der an der Goldsmiths University of London lehrt und mehrere wichtige Publikationen zum Ersten Weltkrieg vorgelegt hat, analysiert die Gräuel der russischen Armee an der Bevölkerung Ostpreußens im ersten Kriegsjahr und ihre Wahrnehmung durch die Zeitgenossen und die Forschung.

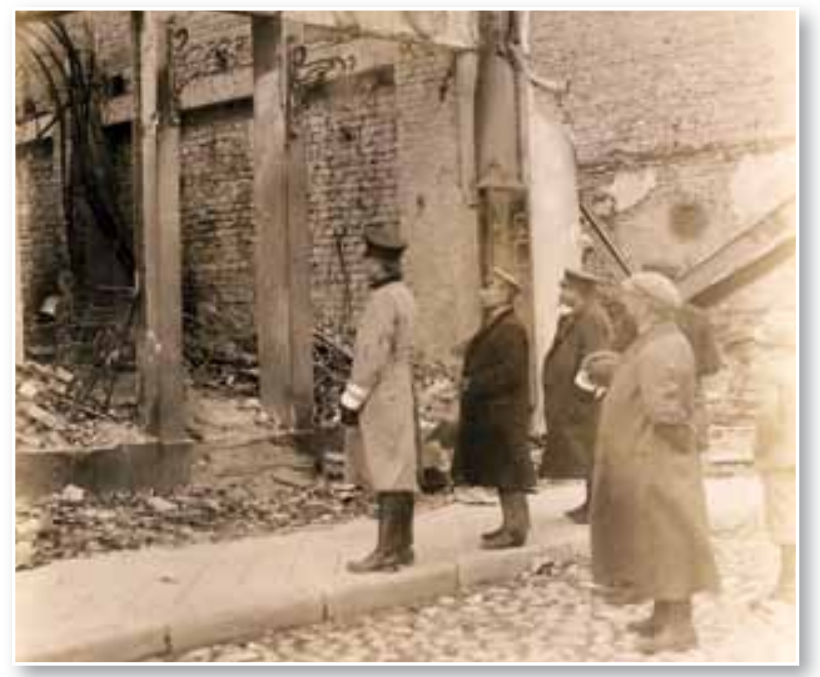

Abb. 2: Schadensbegutachtung in Ostpreußen
30.10.2014: Manfried Rauchensteiner: Der Erste Weltkrieg und das Ende der Habsburgermonarchie 1914-1918.

In Darstellungen und Gesprächen über den Ersten Weltkrieg nimmt Österreich-Ungarn meist nur eine Nebenrolle ein. Dabei nahm der "Große Krieg", der das Ende der Habsburgermonarchie herbeiführte, hier seinen Anfang. Manfried Rauchensteiner, Professor an der Universität Wien, geht daher der Frage nach, welche Rolle Österreich-Ungarn bei der Entfesselung des Ersten Weltkrieges spielte und wie es zum Zerfall des Habsburgerreiches kam.

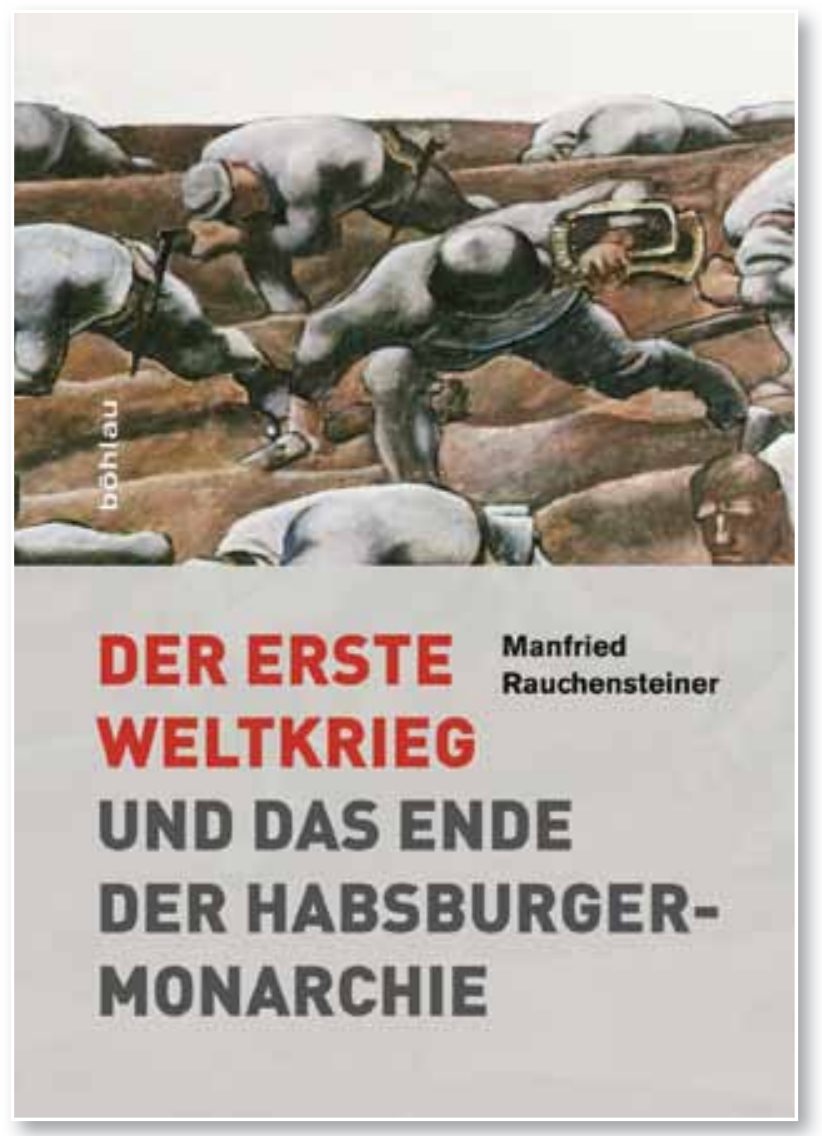

Abb. 3: Manfried Rauchensteiner: Der Erste Weltkrieg und das Ende der Habsburgermonarchie

2.12.2014: Podiumsdiskussion mit Dr. Gerhard P. Groß und Prof. Dr. Gerhard Hirschfeld: „Warum die Soldaten Weihnachten 1914 nicht zu Hause waren. Kann man eine Militärgeschichte ohne Krieg schreiben?"

Gerhard P. Groß, Militärhistoriker am Zentrum für Militärgeschichte und Sozialwissenschaften der Bundeswehr, sowie Gerhard Hirschfeld, prominenter Vertreter der Kulturgeschichte des Ersten Weltkrieges, ziehen zum Ende des WeltkriegsGedenkjahres 2014 Bilanz zur Forschung über und die Erinnerung an den Ersten Weltkrieg. 


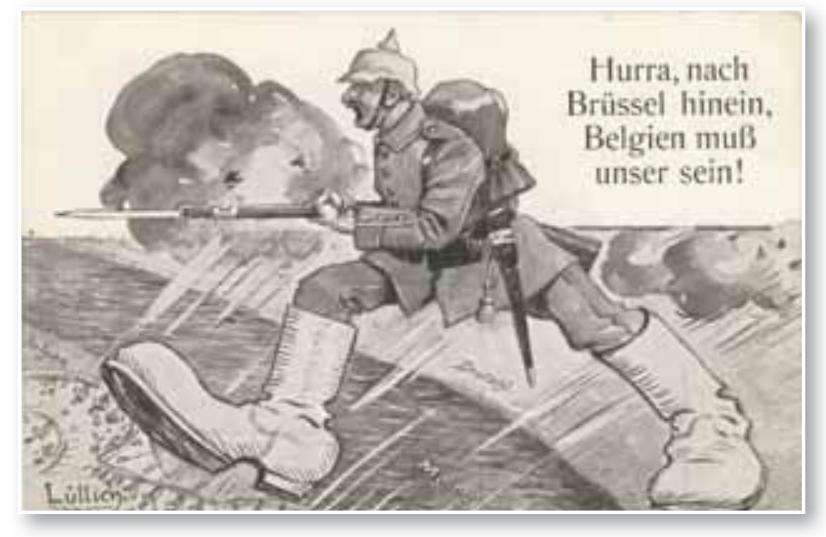

Abb. 4: Deutsche Propaganda-Postkarte 1914

Die Vorträge finden, wenn nicht anders angegeben, um 18 Uhr im Vortragsraum der WLB statt.

\section{Plakate}

Plakate waren im Ersten Weltkrieg ein wichtiges Medium, mit dem sich Regierung und Behörden an die Bevölkerung richteten. Zu unterscheiden sind illustrierte Plakate und Schriftplakate. Illustrierte Plakate sollten beim Betrachter Emotionen wecken und die Bevölkerung für den Krieg mobilisieren, z.B. im Rahmen einer Spendenaktion (Abb. 5).

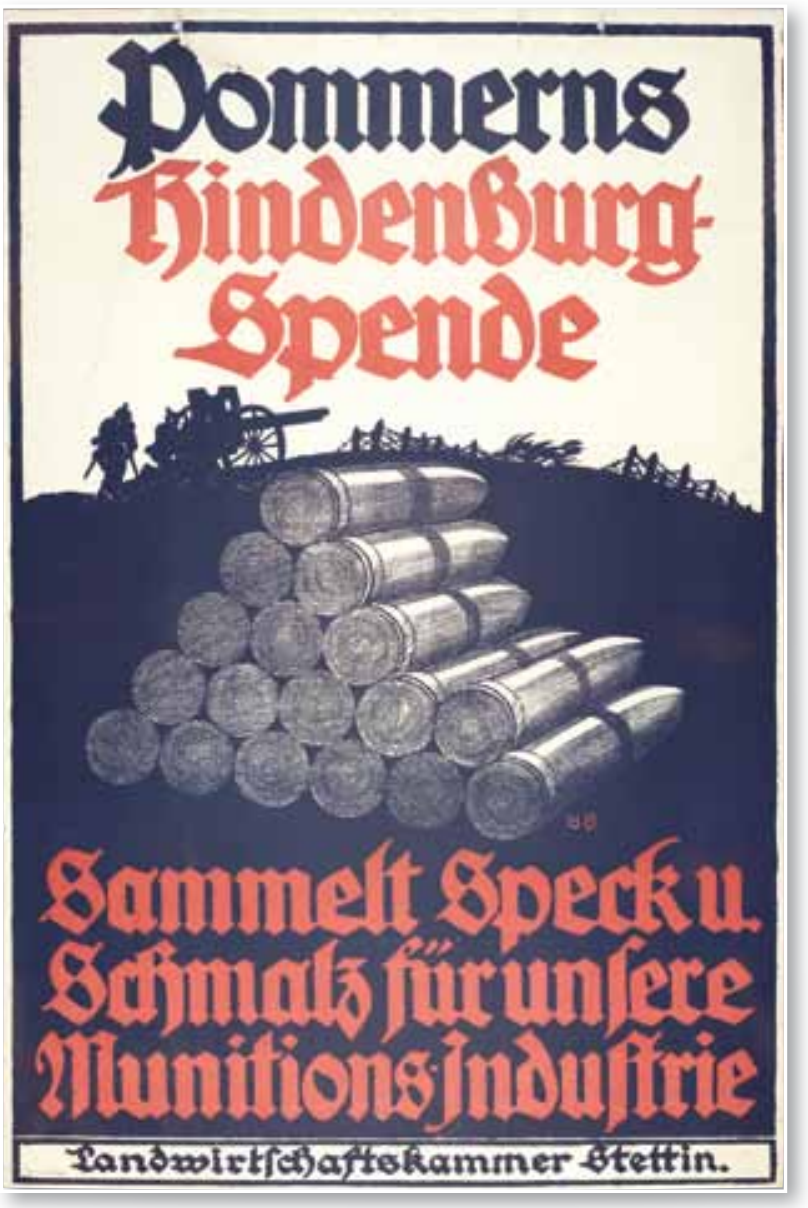

Abb. 5: Illustriertes Plakat: Pommerns Hindenburg-Spende, 1916
Schriftplakate hingegen dienten in erster Linie der Information. So genannte Maueranschläge wurden z.B. von den Militär- und Besatzungsbehörden in den von Deutschland besetzten Gebieten genutzt, um Verordnungen bekanntzugeben (Abb. 6).

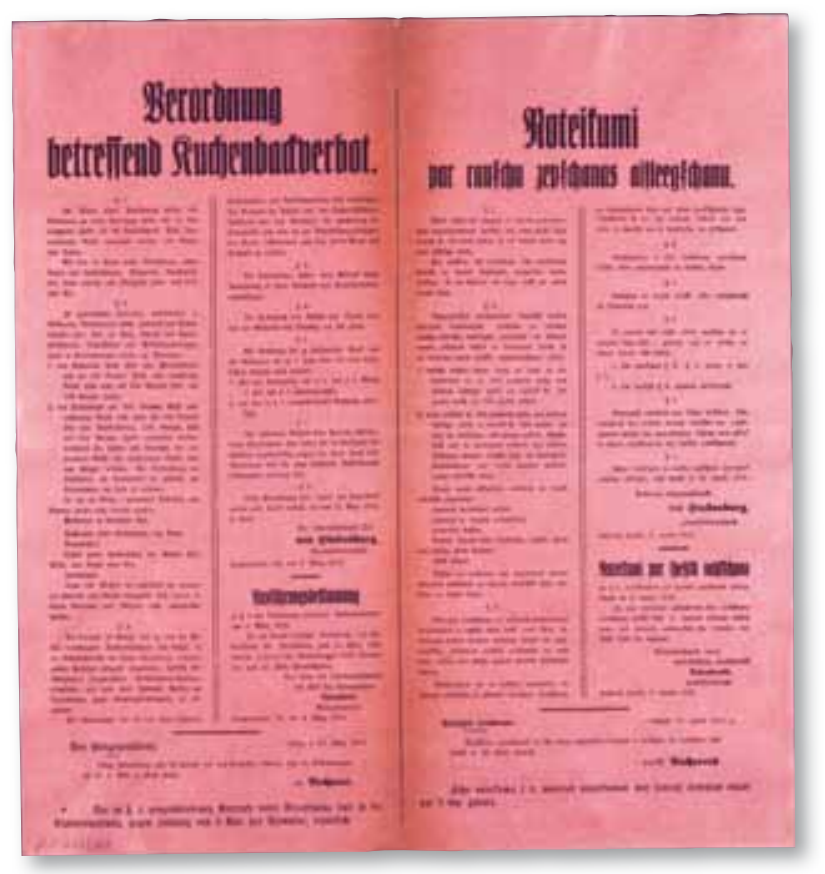

Abb. 6: Maueranschlag: Verordnung betreffend Kuchenbackverbot, Libau (Lettland) 1916

Die BłZ verfügt über eine umfangreiche Plakatsammlung, die über die Plakat-Datenbank ${ }^{3}$ erschlossen ist. Für das Themenportal wurde ein Ausschnitt angefertigt, der sich auf die ca. 4.300 Plakate aus der Zeit des Ersten Weltkrieges konzentriert. Neben Plakaten aus Deutschland, die den Schwerpunkt des Bestandes bilden, sind auch Werke aus Belgien, Bulgarien, Frankreich, Österreich, Russland und den USA zu sehen. Viele illustrierte Plakate sowie alle Schriftplakate werden mit Abbildungen in der Datenbank angezeigt. Die Schriftplakate wurden 2013 in hoher Auflösung digitalisiert, damit sie auch online lesbar sind. Recherchiert werden kann unter anderem nach dem Künstler oder Herausgeber, dem Titel, dem Erscheinungsjahr oder nach Schlagwörtern.

\section{Bücher und Broschüren}

Die BfZ sammelte insbesondere in den ersten Jahrzenten ihres Bestehens, als sie noch den Titel „Weltkriegsbücherei" trug, sehr breit die umfangreiche Literatur zum Ersten Weltkrieg. Neben der politik- und militärgeschichtlichen Literatur wur- 
den die Erinnerungsliteratur der Kriegsteilnehmer, religiöse Schriften, literarische Texte und sogar Kinder- und Kochbücher angeschafft. Die Zeit des Weltkrieges sollte in allen Facetten dokumentiert werden. Durch die internationalen Kontakte der Firma Franck konnte auch ausländisches Schrifttum umfangreich erworben werden. Die von 1915 bis 1933 beschaffte Literatur war von den Zerstörungen während des Zweiten Weltkrieges kaum betroffen. Dadurch ist ein einmaliger historischer Buchbestand fast vollständig erhalten.

Aus dieser vielfältigen Sammlung hat die BfZ im Jahr 2013 ca. 100 Bücher und Broschüren digitalisiert. Weitere 250 Werke sollen demnächst folgen. Die digitalisierten Werke decken verschiedene Aspekte des Ersten Weltkrieges exemplarisch ab und geben Einblick in das militärische, politische, wirtschaftliche, soziale und kulturelle Leben während des Krieges. Neben deutschen Titeln wurden auch Werke aus dem Ausland einbezogen, die in deutschen Bibliotheken bisher nicht online zugänglich sind. Generell wurden Werke ausgewählt, die für Forschung und Lehre eine wichtige Ergänzung zu bestehenden Digitalen Sammlungen bilden. Die Bereiche Propaganda, Leitfäden und Württembergica werden im Folgenden exemplarisch vorgestellt.

Die Digitalisate sind im Online-Katalog der WLB sowie überregional über den SWB Online-Katalog bzw. über den Karlsruher Virtuellen Katalog (KVK) nachgewiesen. Der Zugang über das Themenportal bedeutet für den Nutzer ein erweitertes Angebot. Er kann dort in der thematisch einschlägigen Titelliste browsen.

\section{Propaganda}

Der Erste Weltkrieg war auch ein Krieg der Propaganda. Neben Flugblättern und Plakaten wurden zahlreiche Bücher und Broschüren zu diesem Zweck publiziert. Wenig subtil, dafür recht plastisch sind in der Regel Publikationen, welche die vorgeblichen Missetaten der Feinde und die angeblichen Missstände in den Feindstaaten thematisieren. So publizierte die nationalistisch-konservative Deutsche Vaterlandspartei z.B. 1918 über 100 Schmäh-

(4) Gegen England. 125 Merksprüche entnommen den im Februar 1918 eingereichten 1178 Merksprüchen für das Preisausschreiben des Landesvereins der Deutschen Vaterlands-Partei für Hamburg, Hamburg [1918].

(5) Kainradl, Leo: Unsere Feinde. Ein Kriegsbilderbuch in Verwandlungs figuren, Esslingen / München 1914 sprüche gegen England, die im Zuge eines Preisausschreibens gesammelt wurden. ${ }^{4}$

Auch vor Kindern machte die Propaganda nicht Halt. In eigens geschaffenen Kriegs-Kinderbüchern wurden zum Teil spielerische Elemente integriert. So enthält die Broschüre „Unsere Feinde“ Portraits von Soldaten der Entente, die jeweils die nationalen Stereotype abbilden (Abb. 7). Der russische Soldat trägt beispielsweise neben Mantel, Pelzmütze und Wodkaflasche eine Knute. Die Figuren sind in Kopf-, Mittel- und Fußteil unterteilt, die sich unabhängig voneinander umklappen lassen. Dadurch konnten die Kinder rätseln, welche Attribute zusammengehören oder sich ihre Feinde nach Wunsch selbst zusammenstellen. ${ }^{5}$

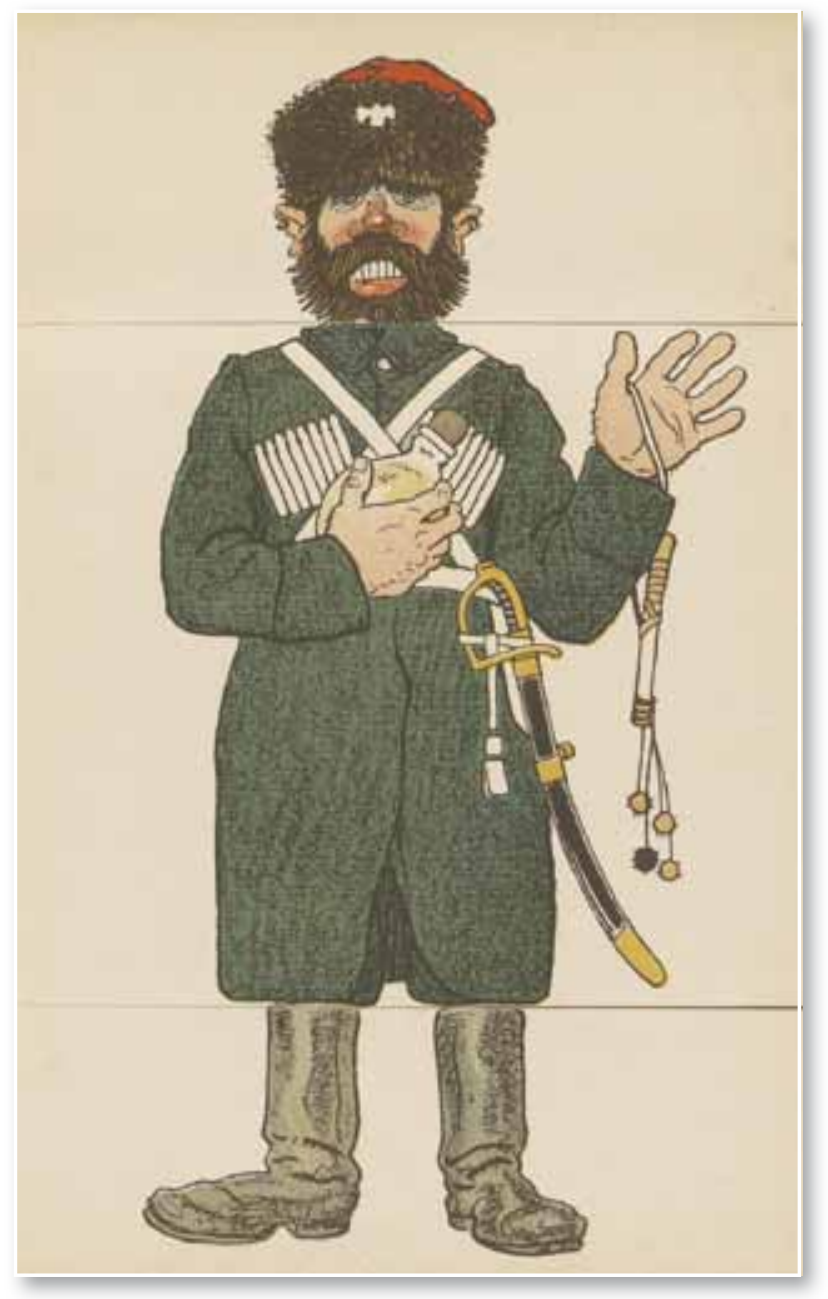

Abb. 7: Der "Russe“ in: Unsere Feinde, 1914

\section{Leitfäden für das Soldatenleben}

Nach Kriegsbeginn waren Millionen von Soldaten mit einer ganz neuen Welt konfrontiert: Sie mussten sich in einem industrialisierten, hochtechnisierten Krieg zurechtfinden, in dem jeder Fehler tödlich 
enden konnte. Verlage und militärische Dienststellen versorgten die Soldaten sowie die Bevölkerung daher mit allerlei Informationen zur modernen Kriegsführung. ${ }^{6}$

Im Zuge des Vormarsches 1914/1915 besetzten deutsche Truppen Belgien, Teile Nordfrankreichs, Russisch-Polen sowie Litauen und Kurland. Die meisten Besatzungssoldaten waren zum ersten Mal in ihrem Leben im Ausland. Um die Orientierung zu erleichtern und um die Moral der Truppe zu erhöhen, gaben die deutschen Besatzungsbehörden Führer durch wichtige Städte wie Brüssel, Kowno oder Warschau (Abb. 8) ${ }^{7}$ heraus. Diese Reiseführer sind heute aus kulturgeschichtlicher Sicht interessant, weil sich aus innen der Blick der Besatzungsverwaltungen auf die besetzten Städte, deren Bewohner und deren Geschichte ablesen lässt.

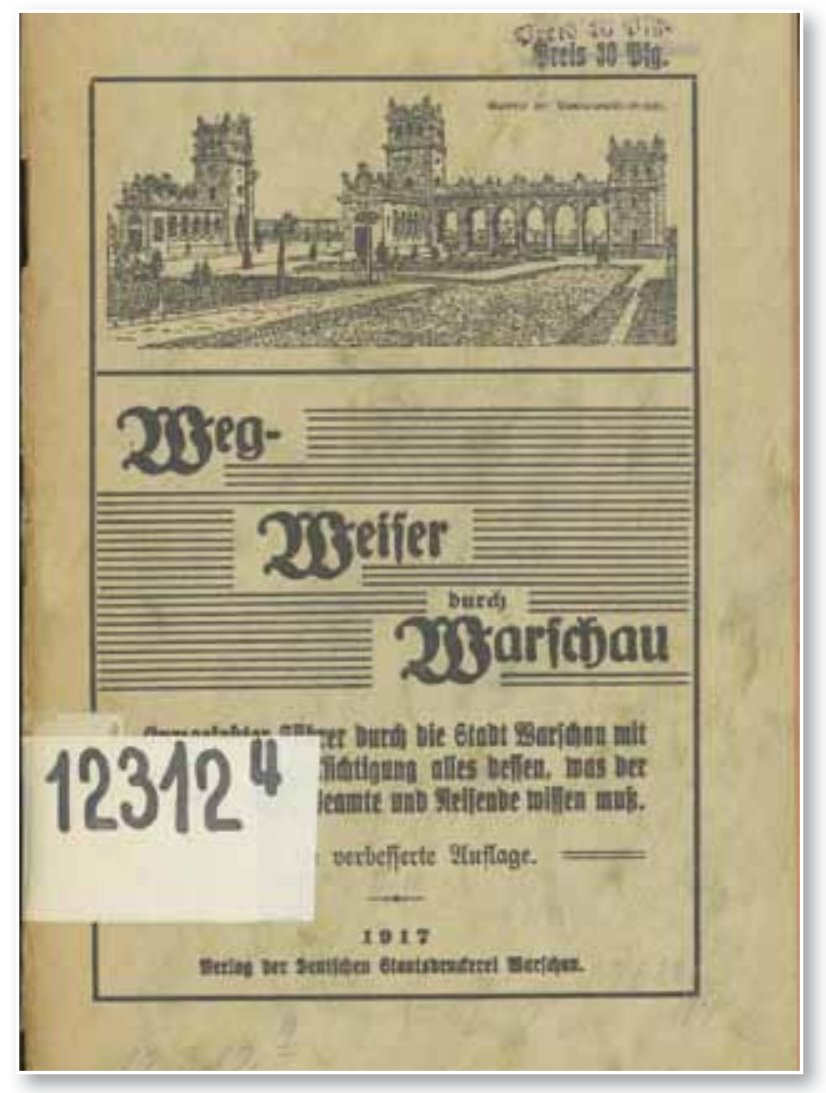

Abb. 8: Weg-Weiser durch Warschau, 1917

\section{Württembergica}

Ein Teil der bisher digitalisierten Werke befasst sich mit Württemberg in den Jahren 1914-1919. Viele württembergische Orte gaben nach dem Krieg Chroniken heraus, ${ }^{8}$ in denen sie zum einen der Einwohner gedachten, die am Krieg teilgenommen hatten oder gefallen waren. Zum anderen beschreiben viele Kriegschroniken die Geschehnisse und
Entwicklungen in den jeweiligen Städten während des Krieges. Diese Werke bieten die Möglichkeit, die Auswirkungen des Ersten Weltkrieges in einer bestimmten Region nachzuvollziehen. Auch die regionale Ausformung von Trauer und Erinnerung lassen sich untersuchen.

Das Kriegserlebnis der württembergischen Soldaten versuchten Truppengeschichten festzuhalten, die in den 1920er und 1930er Jahren in großer Zahl erschienen. Einen umfassenden Überblick über die Teilnahme württembergischer Truppen am Krieg gibt das mächtige Werk des Weltkriegsgenerals Otto von Moser "Die Württemberger im Weltkriege". ${ }^{9}$ Mit mehr als 700 Seiten und zahlreichen Illustrationen bildet es ein wichtiges Nachschlagewerk für die Landesgeschichte. In zahlreichen Kapiteln wird die Geschichte der verschiedenen Einheiten nachverfolgt, in denen württembergische Soldaten dienten. Durch die Indexierung der einzelnen Kapitel ist es möglich, im Digitalisat gezielt einen bestimmten Teil des Buches anzusteuern (Abb. 9). Dadurch entfällt das Blättern im wenig handlichen Original. Für 2014 ist überdies geplant, die Truppengeschichten der verschiedenen württembergischen Einheiten zu digitalisieren.

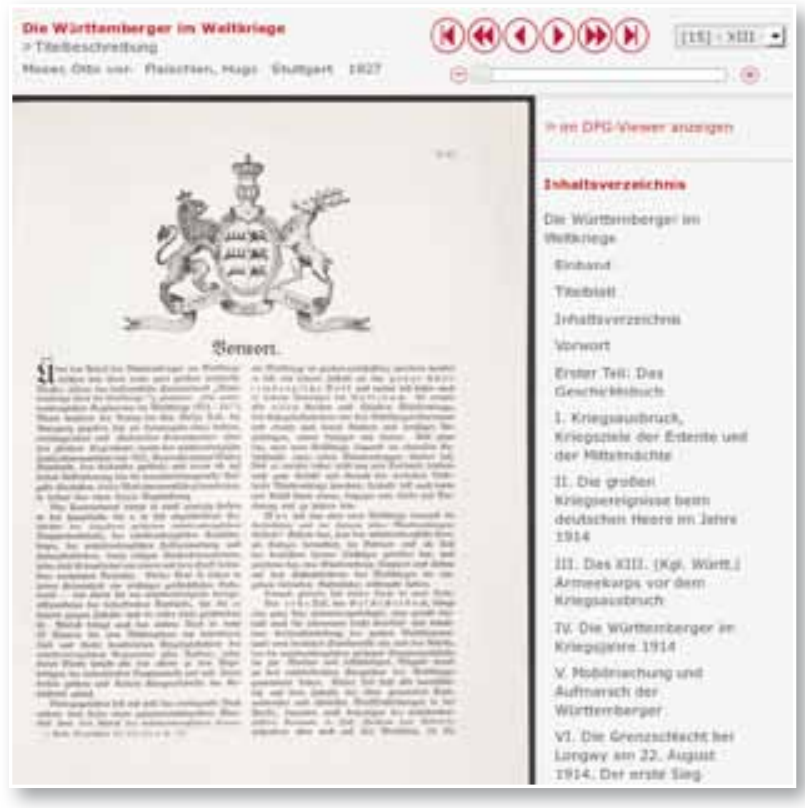

Abb. 9: "Die Württemberger im Weltkriege" als Digitalisat mit ansteuerbaren Kapiteln

(6) Siehe z.B. Leitfaden für das Maschinengewehr 08/15, Berlin 1917.

(7) Weg-Weiser durch Warschau. Kurzgefaßter Führer durch die Stadt Warschau mit besonderer Berücksichtigung alles dessen, was der deutsche Soldat, Beamte und Reisende wissen muß, Warschau 1916.

(8) Z.B. Biberach, Bruchsal, Ebingen, Ellwangen, Heilbronn, Mengen, Oberndorf a.N., Stuttgart, Weingarten.

(9) Die Württemberger im Weltkriege. Ein Geschichts-, Erinnerungsund Volksbuch, bearb. von Otto v. Moser. Mit Skizzen von Hugo Flaischlen und Kriegsgliederungen von der Reichsarchivzweigstelle Stuttgart, Stuttgart 1927 


\section{Kriegszeitungen}

Das Themenportal bietet auch eine Übersicht über mehr als 70 von verschiedenen Institutionen digitalisierten Feld- und Besatzungszeitungen der BfZ aus dem Ersten Weltkrieg. Der Erste Weltkrieg war der erste Krieg, in dem Soldaten selbst in großer Zahl eigene Zeitungen erstellten. Diese dienten dazu, den Frontalltag aufzuheitern und das Erlebte zu verarbeiten. Auch wollte man eine alternative Informationsquelle zu den offiziellen Berichten oder den Zeitungen aus der Heimat haben, die insbesondere an der Ostfront schwer zu beschaffen waren. Nach und nach gerieten die meisten Feldzeitungen allerdings unter Kontrolle der Militärbehörden, die die Zeitungen für ihre Durchhaltepropaganda nutzten. Die deutschen Behörden in den besetzten Gebieten gaben eigene Zeitungen heraus. Die Zeitungen gewähren interessante Einblicke in das politische, wirtschaftliche und kulturelle Leben in den besetzten Territorien, zumal der Großteil der Archivakten sich nicht erhalten hat.

Von der Startseite des Themenportals gelangt man zunächst auf eine Überblicksseite, auf der die mehr als 70 aufgeführten Zeitungen in alphabetischer Reihenfolge aufgelistet sind. Von hier aus verweisen die Titel der Zeitungen auf Unterseiten, welche die analogen Bestände der BfZ zu einzelnen Zeitungen aufzeigen. Links lenken außerdem auf externe Digitalisate anderer Institutionen, z.B. der UB Heidelberg. Diejenigen Digitalisate, welche die Firma Adam Matthews aus den Beständen der $\mathrm{BfZ}$ erstellt hat, sind hier für registrierte Nutzer der WLB ebenfalls zugänglich. Langfristig sollen die Bestände einschließlich der Gefangenenzeitungen sämtlich in den überregionalen Katalogen ZDB und SWB erschlossen und mit den Digitalisaten verlinkt werden.

Darüber hinaus bietet die WLB ihren registrierten Nutzern als einzige Bibliothek in Deutschland einen Zugriff auf die Datenbank "Trench Journals and Unit Magazines of the First World War" der Firma Proquest an. Mit über 1.500 Titeln aus zahlreichen Ländern steht damit die weltweit wohl größte digitale Sammlung von Kriegszeitungen zur Verfügung. Vor allem unter den Zeitungen der Entente-Truppen sind hier seltene Lager- und Lazarettzeitungen zu finden. Der Großteil der Zeitungen stammt von der Westfront. Die Sammlung enthält jedoch auch
Titel von der Ostfront, der Alpenfront und aus dem Nahen Osten. Die Datenbank erlaubt unter anderem Volltextsuche, PDF-Download, Druck und den Export in Literaturverwaltungsprogramme. Sie kann entweder über das Themenportal Erster Weltkrieg (unter „Kriegszeitungen“), die Digitale Bibliothek oder den Online-Katalog der WLB angesteuert werden.

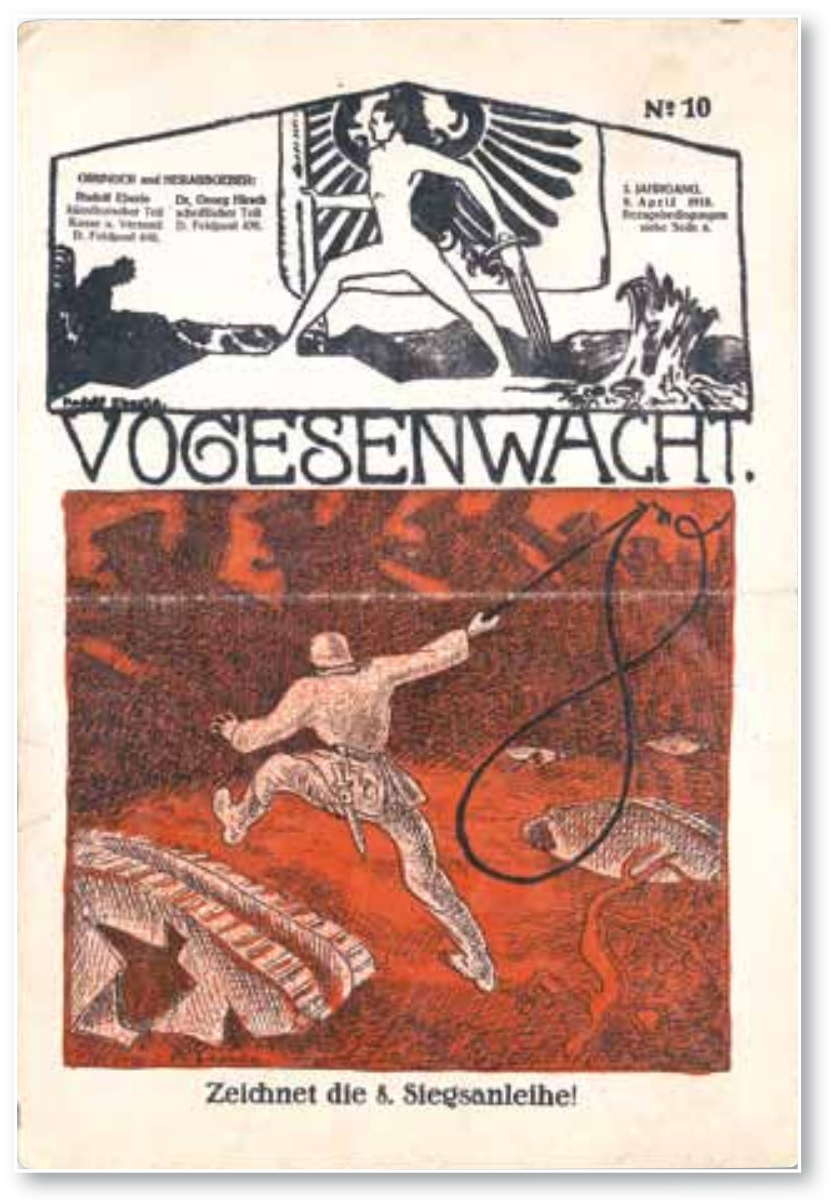

Abb. 10: Feldzeitung "Vogesenwacht" vom 9. April 1918

\section{Tagebücher und Briefe}

Truppengeschichten liefern Informationen über die militärischen Operationen und Geschehnisse des Krieges. Auch geben sie Auskunft über die Gedenkkultur der Truppen. Über das individuelle Kriegserlebnis der Soldaten erfährt der Leser in diesen Werken hingegen wenig. Hierfür sind Tagebücher und Briefe wesentlich besser geeignet. Denn jeder Zeitzeuge nahm den Krieg unterschiedlich wahr und stellte inn in solchen Lebensdokumenten anders dar, abhängig von Herkunft, Alter, Stellung und Schreibstil.

Die Lebensdokumentensammlung der BfZ enthält ca. 50.000 Feldpostbriefe, Tagebücher und Erinne- 
rungen von Kriegsteilnehmern des Ersten Weltkriegs. Ausgewählte Beispiele wurden digitalisiert und transkribiert. Um den Zugang zu den häufig in Kurrentschrift verfassten Tagebüchern zu erleichtern, wurden Exemplare ausgewählt, für die auch eine Transkription sowie weitere Materialien vorliegen. Die Tagebücher werden nun sowohl als Faksimile des Originals als auch als Transkription angeboten, angereichert um eine Einleitung und Fotos. Die präsentierten Tagebücher von Theodor Zuhöne (Abb. 11), einem 37jährigen Arzt aus Damme, und von Otto Borggräfe, einem 19jährigen Kriegsfreiwilligen aus Oldenburg, zeigen sehr deutlich die unterschiedliche Kriegserfahrung der Zeitgenossen. Schon seit längerem stehen außerdem die Briefe und Tagebücher des Oberleutnants August Dänzer zur Verfügung, der 1916/1917 am Rumänienfeldzug teilnahm. Auszüge aus seinem Nachlass wurden bereits 2009 im Rahmen eines Editionsprojektes der Universität Potsdam transkribiert, digitalisiert und mit Hintergrundinformationen angereichert.

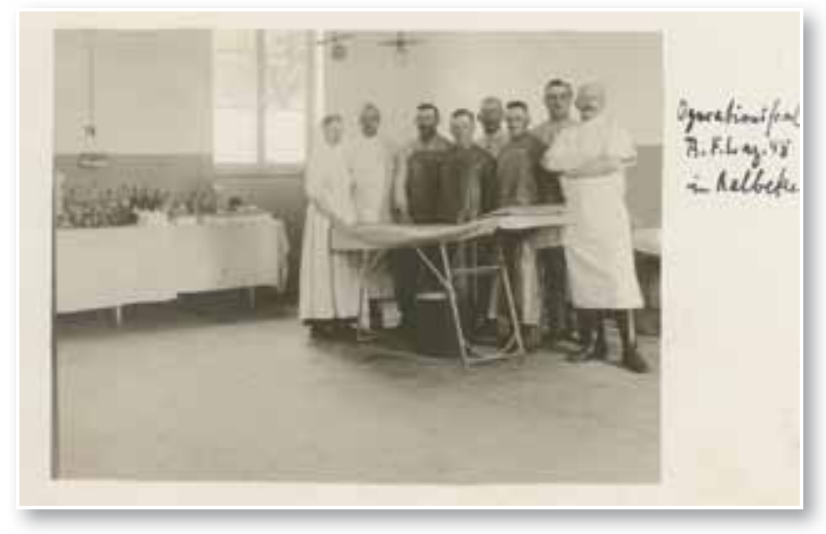

Abb. 11: Dr. med. Theodor Zuhöne (rechts) im Reserve Feldlazarett 48 (La Neuville)

Weitere Tagebücher sollen 2014 digitalisiert und im Bibliothekskatalog nachgewiesen werden. Neben den unveröffentlichten Unikaten sollen 2014 außerdem 150 Tagebücher und Feldpostsammlungen digitalisiert werden, die noch während des Ersten Weltkrieges oder in den Jahren unmittelbar danach erschienen. Hierbei sollen neben deutschen Werken auch englische und französische Publikationen einbezogen werden.

\section{Rationierungsmarken}

Der angespannten Versorgungslage während des Krieges versuchten die Länder- und Kommunalverwaltungen mit Rationierungsmaßnahmen entgegenzuwirken. Zu diesem Zwecke wurden an die
Bevölkerung Rationierungsmarken für Lebensmittel, Energie und andere Konsumgüter ausgeteilt.

Die BłZ verfügt über eine Sammlung von Rationierungsmarken mit mehr als 5.000 unterschiedlichen Exemplaren aus dem Ersten Weltkrieg. Die Marken stammen aus verschiedensten Städten und Gemeinden des Deutschen Reichs (Abb. 12) sowie aus dem europäischen Ausland. Auch aus den besetzten Gebieten haben sich Marken erhalten. Der gesamte Bestand ist nun über eine Datenbank online recherchierbar. Gesucht werden kann unter anderem nach den rationierten Gütern, nach Ort, Region oder Land, nach der Sprache sowie nach dem Jahr.

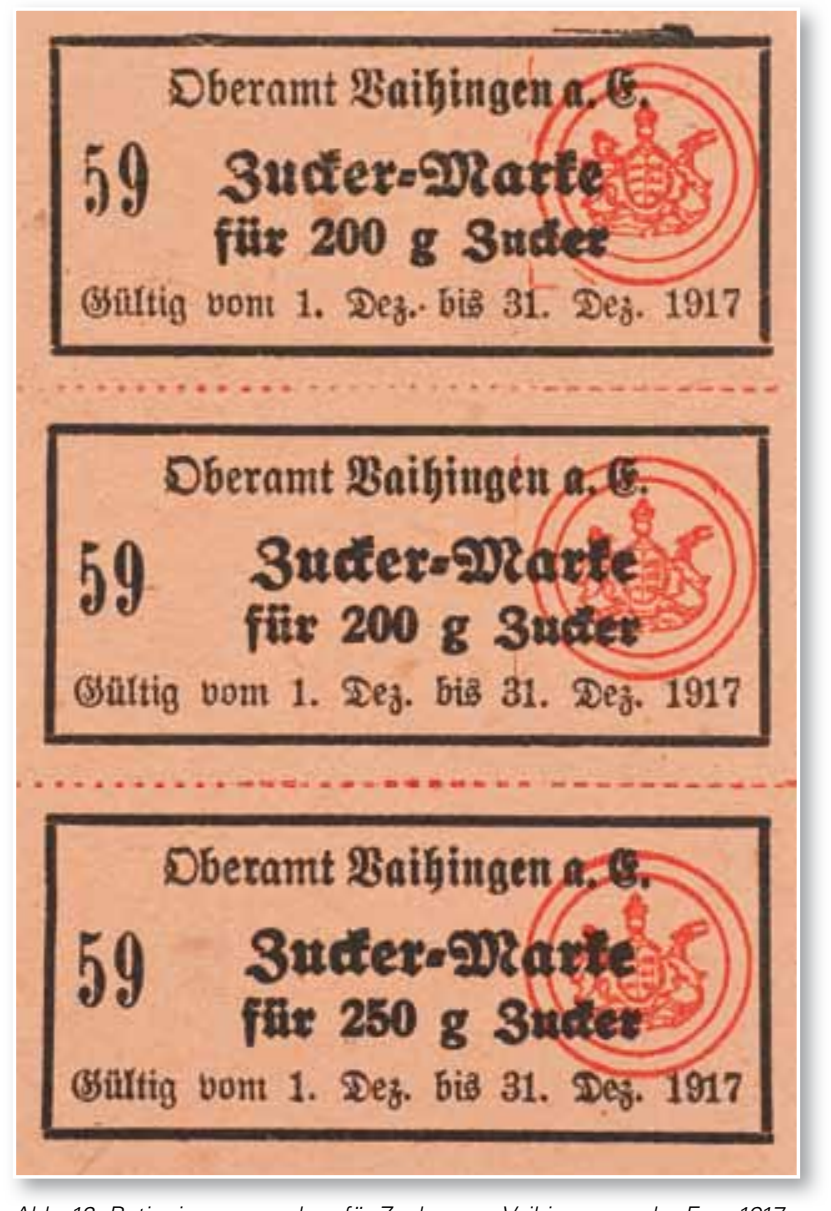

Abb. 12: Rationierungsmarken für Zucker aus Vaihingen an der Enz, 1917

\section{Digitale Dokumentensammlungen für Nutzer der WLB}

Die Firma Adam Matthews hat Zeitungen, Flugblätter, Bücher, Broschüren, Plakate und Postkarten zum Ersten Weltkrieg aus den Sammlungen der BfZ digitalisiert. Diese Materialien wie auch Digitalisate aus anderen namhaften internationalen Einrichtungen wie dem Imperial War Museum sind 
hier online zugänglich. Die Volltextdatenbank, die auch Tagebücher, Briefe, Fotos, Karten und persönliche Gegenstände enthält, wird in Deutschland nur von der WLB angeboten.

Der Nutzer kann in der Datenbank über Titel-, Schlagwort- oder Volltextsuche recherchieren. Außerdem sind die Zeitungsartikel einzeln ansteuerbar. Ebenso wie "Trench Journals and Unit Magazines of the First World War" ist "The First World War" von Adam Matthews sowohl über das Themenportal als auch über die Digitale Bibliothek sowie den Online-Katalog der WLB zugänglich. Die Datenbanken können über Login auch außerhalb der Bibliothek genutzt werden.

\section{Resonanz}

Das Themenportal wurde mittlerweile auf verschiedenen Wegen der Öffentlichkeit präsentiert. Von den Websites von Clio-Online, ${ }^{10}$ der Stadt Stuttgart, ${ }^{11}$ von Kriegssammlungen.de, ${ }^{12}$ Volksbund Deutsche Kriegsgräberfürsorge e. V., ${ }^{13}$ der Landeszentrale für politische Bildung Baden-Württemberg $^{14}$ sowie vom Portal Militärgeschichte ${ }^{15}$ wird auf das Themenportal verwiesen. Am 25. Oktober 2013 stellte es Christian Westerhoff außerdem auf einer Tagung zum Ersten Weltkrieg in Oldenburg vor. Über die Tagung, das Themenportal sowie die Tagebücher Zuhöne und Borggräfe berichtete die regionale Presse ${ }^{16}$ und das Fachportal HSozKult ${ }^{17}$. Auch für 2014 ist geplant, das Themenportal auf verschiedenen Veranstaltungen zu präsentieren.

Auf der Konferenz „Unlocking Sources", die am 30./31. Januar 2014 stattfand, ${ }^{18}$ hob der Historiker Sönke Neitzel zum einen den großen Wert von Tagebüchern und Briefen für die Forschung zum Ersten Weltkrieg hervor. Zum anderen betonte er aber auch, wie wichtig es ist, dass Faksimile-Digitalisate mit Abschriften und Kontextinformationen angereichert werden, wie dies auf dem Themenportal Erster Weltkrieg der Fall ist.

Positive Rückmeldungen für das Themenportal gibt es auch von den Personen, die der BfZ Tagebücher samt Zusatzmaterialien überlassen haben und die Erlaubnis zur Online-Publikation erteilten. Speziell die Präsentation der Tagebücher mit Transkription und Kontextinformationen wurde sehr gut aufgenommen. In weiteren Fällen war die Aussicht auf die Netzpublikation für die Sponsoren sogar ein Anreiz, ihre Materialien der Sammlung der BfZ zu übergeben.

Christian Westerhoff
(10) http://www.erster-weltkrieg.clio-online.de

(11) http://www.stuttgart.de/weltkrieg

(12) https://www.kriegssammlungen.de

(13) http://100-jahre-erster-weltkrieg.eu/internetangebote/deutschland.html

(14) http://www./pb-bw.de/ersterweltkrieg.htm

(15) Westerhoff, Christian / Renz, Irina: Die Sammlungen und Aktivitäten der Bibliothek für Zeitgeschichte im Weltkriegs-Gedenkjahr 2014/2015. Artikelserie 100 Jahre Erster Weltkrieg - 100 Jahre Bibliothek für Zeitgeschichte, hrsg. v. Christian Westerhoff. In: Portal Militärgeschichte: 30. Dezember 2013, URL: http://portal-militaergeschichte. de/westerhoff_renz_sammlungen (3.3.2014).

(16) Tagebuch eines Dammer Militärarztes: Historiker hat die Aufzeichnungen aus dem Ersten Weltkrieg digitalisiert. In: Oldenburgische Volkszeitung: 24.10.2013. Erinnerungen an den Krieg aus unterschiedlichen Blickwinkeln. In: Heimatblätter, Beilage zur "Oldenburgischen Volkszeitung": 14.12.2013, S. 52.

(17) Tagungsbericht 1. Tagung zur Oldenburgischen Regionalgeschichte: Oldenburg im Ersten Weltkrieg. 25.10.2013, Oldenburg. In: H-Soz-uKult: 29.11.2013, URL: http://hsozkult.geschichte.hu-berlin.de/ tagungsberichte/id=5119 (3.3.2014)

(18) http://www.europeana-collections-1914-1918.eu/unlocking-sources/ 